\title{
Performance of Two-Hop DS-CDMA Systems Using Amplify-and-Forward Protocol over Different Fading Channels
}

\author{
Nuri Kapucu ${ }^{1}$, Mehmet Bilim ${ }^{1}$, Ibrahim Develi ${ }^{1}$ \\ ${ }^{I}$ Department of Electrical and Electronics Engineering, Erciyes University, \\ 38039, Kayseri, Turkey \\ nurikapucu@erciyes.edu.tr
}

\begin{abstract}
This study analyses the performance of directsequence code division multiple access (DS-CDMA) based on two-hop amplify-and-forward protocol over Weibull symmetric fading channels as well as Rayleigh/Rician, Rician/Rayleigh asymmetric fading phenomenas. We investigate the bit-error rate (BER) of the considered system using multiple relays by considering the effect of Weibull fading parameter and Rician $K$ factor on the system performance. Our simulation results demonstrate the positive impacts of the value of fading parameter, Rician $K$ factor and increasing number of relay nodes on BER performance. It is also confirmed that the Rician $K$ factor is more effective on the system performance over Rician/Rayleigh fading channels in comparison with Rayleigh/Rician fading environment.
\end{abstract}

Index Terms-Two-hop relaying, DS-CDMA, fading channels, amplify-and-forward.

\section{INTRODUCTION}

Direct-sequence code division multiple access (DSCDMA) which is one of the wireless and mobile communication techniques, has an important role and is commonly used in our daily communication technology. The main objective of DS-CDMA technique is increasing bandwidth and providing a secure communication in a frequency limited system [1]-[6]. In some cases, there may not be possible to communicate between transmitter and receiver due to path loss, shadowing and fading effects. In this cases, two-hop relaying, in which a relay node is used for the transmission of source information, can be a useful alternative to maintain the communication in DS-CDMA systems due to its connectivity. Two hop relaying is an effective method to achieve the high transmission rates and extend the coverage range. In two-hop transmissions, two different techniques can be employed such as amplify-andforward (AF) and decode-and-forward (DF) relaying. In DF relaying method, relay sends an estimation of source information to destination. On the other hand, the relay amplifies the source information and sends the amplified source information to the destination in AF method [7].

Manuscript received 24 December, 2014; accepted 29 May, 2015.

This work was supported by the Scientific and Technological Research Council of Turkey (TUBITAK) under project grant 114E242. The authors thank to the Scientific Research Projects Coordinating Office of Erciyes University (EU-BAP, Project No: FDK-2014-5166).
Several papers have analysed performance of DS-CDMA systems based on cooperative diversity in which direct transmission is considered in addition to two-hop relaying over different fading channels such as Rayleigh, Nakagami$m$ and frequency selective fading channels [8]-[17]. In [8], the authors investigated the bit-error rate (BER) performance of DS-CDMA systems by applying cooperative DF relaying technique over Rayleigh faded channels. A joint successive interference cancellation (SIC) detection method with a multi-relay selection algorithm was proposed for the uplink of cooperative DS-CDMA systems in [9]. Li et al. evaluated the capacity of cooperative DS-CDMA systems based on both AF and DF methods in Rayleigh fading channels [10]. The authors in [11] considered power allocation problem for DS-CDMA system based on cooperative communications when the transmission channels are subject to Rayleigh distribution. In another work, the performance of AF/DF based DS-CDMA systems are investigated with use of non-orthogonal spreading codes in Rayleigh fading environments [12]. The study presented in [13] gives the evaluation of downlink transmission in a cooperative DS-CDMA in Nakagami- $m$ distributed channels by assuming preprocessing at the receiver. Mehemed et al. [14] evaluated the outage probability of AF cooperative diversity for DS-CDMA systems under Nakagami- $m$ faded transmission environments. Convolutional coded cooperative DS-CDMA systems are studied over frequency selective fading environments in [15].

In the studies above [8]-[15], cooperative diversity based DS-CDMA systems are investigated under symmetric fading conditions where all channels between cooperating nodes have the same fading distributions. However, symmetric fading environment is not suitable for the real wireless channel characteristics. On the other hand, the channels between cooperating nodes can experience different fading conditions (asymmetric fading conditions) in realistic wireless relay networks. In the literature, there are several studies that are presented the performance of cooperative diversity and two-hop relaying systems without integrating DS-CDMA technique under asymmetric channel conditions [16]-[21]. In [16]-[17], the authors studied the outage probability and bit-error probability of two-hop AF relaying (without DS-CDMA system) protocols when the channels 
are subject to Rayleigh/Rician and Rician/Rayleigh fading cases. Xu et al. [18] examined performance of two-hop AF transmission without DS-CDMA principle in Nakagami$m /$ Rician fading channels. In [19], outage probabiliy of twohop DF relaying scheme was investigated for Rayleigh/Gamma fading conditions. The authors in [19] proposed an analytical expression for the outage probability of the considered cooperation model in their work. Spalevic et al. [20], examined a dual-hop AF relaying transmission model under mixed Rayleigh/Hoyt fading. In [21], the performance of two-hop DF transmission is studied in Rayleigh/Weibull asymmetric environments.

Despite the many recent studies about cooperative DSCDMA systems, to the best of authors' knowledge, none of them has presented the performance of DS-CDMA based on two-hop AF relaying over symmetric and asymmetric fading channels. Motivated by these observations, for the first time, we study DS-CDMA systems based on two-hop AF relaying over symmetric Weibull fading channels that the use of Weibull channel model is recommended by IEEE Vehicular Technology Society Committee on Radio Propagation. In addition to these, we analyse the performance of DS-CDMA by using two-hop AF transmission model over asymmetric fading channels where the channels of two hops experience Rayleigh/Rician and Rician/Rayleigh conditions, respectively. Moreover, we illustrated BER performance of two-hop AF relayed DS-CDMA system for different scenarios. Furthermore, we present the effects of Weibull fading parameter and Rician $K$ factor on the system performance by providing computer simulations.

\section{DS-CDMA SYSTEMS}

Spread spectrum based CDMA, has an important role in our daily life communications. Multiple access systems allow more than one user to share frequency (bandwidth) and time, simultaneously. One of the important CDMA techniques is DS-CDMA. In a traditional DS-CDMA system, each user is separately treated as an information while the other users are considered as interference [1]. In Fig. 1, transmitter of $K$ users DS-CDMA system is shown.

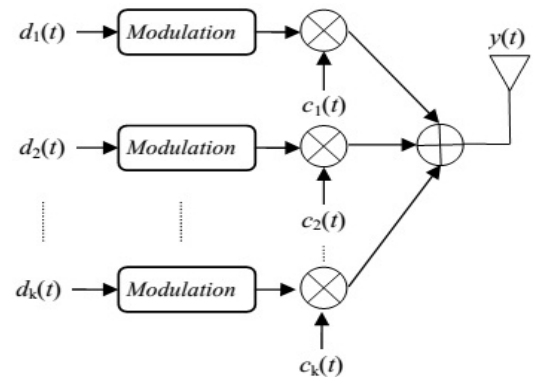

Fig. 1. Block diagram of $K$ simultaneous users DS-CDMA transmitter.

The information of each user, $d_{k}(t)(k=1,2, \ldots, K)$ is modulated and spreaded by the unique spreading code of the $k$ th user, $c_{\mathrm{k}}(t)$, that is different for each user. After that, sum of the $K$ users information, $y(t)$, is transmitted to the receiver. $K$ simultaneous users DS-CDMA receiver block diagram is demonstrated in Fig. 2. At the receiver, $y(t)$ is multiplied by each user's unique spreading code to separate the user information and then, demodulation is applied.
After demodulation process, decision is made to estimate the $k$ th user information $\left(\tilde{d}_{k}\right)$.

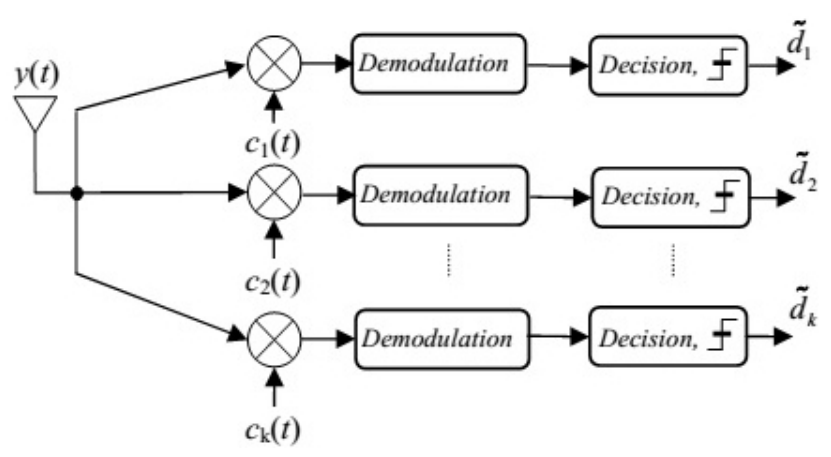

Fig. 2. Block diagram of $K$ simultaneous users DS-CDMA receiver.

\section{System AND CHANNEL ModELS}

\section{A. System Model}

Two-hop transmission is a significant way to maintain the communication when direct transmission is impractical. In this work, AF protocol is considered with DS-CDMA system which was explained in the previous section. Two-hop transmission model with one relay is given in Fig. 3 .

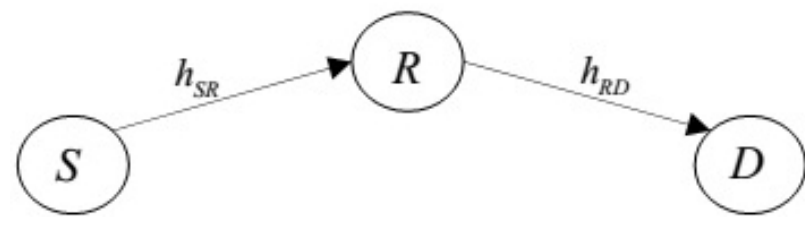

Fig. 3. Illustration of two-hop relaying scheme with one relay.

A source $(S)$ broadcasts the information signal to the destination $(D)$ with the help of a relay $(R)$ in a two-hop system model. $h_{S R}$ and $h_{R D}$ indicate the channel coefficients of $S-R$ and $R-D$ links, respectively. For the considered two-hop AF relaying DS-CDMA system, $S$ node has a DS-CDMA transmitter while $D$ node has a DS-CDMA receiver. In addition to these, $R$ node has DS-CDMA transceiver. In this work, $L=1,2$ and 3 relay nodes are used. The $R$ nodes are assumed to be operating in the half duplex mode. The transmission period consists of two intervals. In the first interval, $S$ transmits user information to the $R$ nodes. The received information at $l$ th $R$ node is given as

$$
r_{l}(t)=\sum_{i=0}^{f-1} \sum_{k=1, k \neq l}^{K} d_{k}(i) C_{k}\left(t-\tau_{k}-i T_{d}\right) \times h_{S R}(i)+n_{l}(t)
$$

where $l=1,2,3, K$ is the number of simultaneous users, $f$ is the frame length, $d_{k}(i)$ is the $i$ th symbol of $k$ th user and $C_{k}$ is the spreading code of the $k$ th user. $T_{d}, T_{c}$ and $\tau_{k}$ represent bit period, chip period and random transmission delay of $k$ th user, respectively. $h_{S R}$ is the channel fading coefficient between $S-R$ and $n_{l}(t)$ is the additive white Gaussian noise (AWGN) which has zero mean and $\sigma_{n}^{2}=N_{0}$ variance.

In the second transmission interval, each relay node 
amplifies the received information according to an amplification factor which can be expressed as [7]

$$
\beta=\sqrt{\frac{E_{b}}{E_{b}\left|h_{S R}\right|^{2}+N_{0}}},
$$

where $E_{b}$ is energy of transmitted signal. $R$ nodes use the amplification factor, $\beta$, and send the amplified source information to the $D$ node. At $D$ node, received signal from $l$ th relay node is written as

$$
r_{D_{l}}(t)=\sum_{i=0}^{f-1} \sum_{k=1}^{K} \sum_{l=1}^{L} r_{l}\left(t-D_{k}-\tau_{r}-i l T_{b}\right) h_{R D_{l}}(i) \beta+n_{D_{l}}(t),(3)
$$

where $\tau_{r}$ is the transmission delay of $l$ th relay, $D_{k}$ indicates the time delay of the second transmission phase, $h_{R D_{l}}$ is the channel coefficient between $l$ th $R-D$ and $n_{D_{l}}(t)$ is AWGN of the $l$ th $R-D$ channel.

At the $D$ node, received signal from each relays are combined with the use of maximum ratio combining (MRC) technique for two-hop system as [22]

$$
r_{D_{M R C}}(t)=\sum_{l=1}^{L} w_{l} r_{D_{l}}(\mathrm{t})
$$

where $L=1,2,3, w_{l}$ is the MRC coefficient of the received signal from the $l$ th $R$ node and $w_{l}=\sqrt{E_{b}} h_{S R D}^{*} / \sigma_{S R D}^{2} . h_{S R D}^{*}$ is the conjugate of the equivalent channel coefficient and $\sigma_{S R D}^{2}$ is the noise variance of the $S-R-D$ link.

\section{B. Channel Models}

This subsection explains the fading models used in the considered two-hop AF relaying with DS-CDMA network. In this study, three different fading scenarios are considered for the system channels.

In scenario $1, S-R$ and $R-D$ channels are subject to Weibull distribution which is the symmetric fading condition. Probability density function (PDF) of Weibull distribution can be given as [23]

$$
\begin{gathered}
f_{\alpha}(\alpha)=c\left(\frac{\Gamma\left(1+\frac{2}{c}\right)}{\Omega}\right)^{c / 2} \times \\
\times \alpha^{c-1} \exp \left[-\left(\frac{\alpha^{2}}{\Omega} \Gamma\left(1+\frac{2}{c}\right)\right)^{c / 2}\right],
\end{gathered}
$$

where $\alpha \geq 0, \Gamma(\cdot)$ is the gamma function, $c$ is fading parameter, $\Omega$ is the scaling parameter and $\alpha$ is the random Weibull variable. In this scenario, the coefficients of $S-R$ and $R-D$ channels are generated according to the PDF in (5).

In scenario $2, S-R$ and $R-D$ links experience non line-ofsight (LoS) Rayleigh fading and LoS Rician fading, respectively which is the asymmetric fading conditions. The PDF of $S-R$ link can be exponentially written according to Rayleigh PDF as [23]

$$
f_{\alpha_{1}}\left(\alpha_{1}\right)=\frac{2 \alpha_{1}}{\Omega} \exp \left(-\frac{\alpha_{1}^{2}}{\Omega}\right)
$$

where $\alpha_{1} \geq 0, \alpha_{1}$ is random Rayleigh variable for $S$ - $R$ link. The PDF of $R-D$ link can be expressed according to Rician distribution as [23]

$$
\begin{aligned}
& f_{\alpha_{2}}\left(\alpha_{2}\right)=\frac{2(K+1) e^{-K} \alpha_{2}}{\Omega} e^{-\frac{(K+1) \alpha_{2}^{2}}{\Omega}} \times \\
& \times I_{0}\left(2 \alpha_{2} \sqrt{\frac{K(K+1)}{\Omega}}\right),
\end{aligned}
$$

where $\alpha_{2} \geq 0, K$ indicates the Rician $K$ factor and $I_{0}(\bullet)$ is the zeroth order modified Bessel function of the first kind.

In the scenario 3, the channels between $S-R$ and $R-D$ links are distributed LoS Rician fading and non LoS Rayleigh fading, respectively. In this scenario, $\alpha_{1}$ is the Rician random variable of $S-R$ link is while $\alpha_{2}$ is the Rayleigh random variable of $R-D$ channel.

In scenarios 2 and 3, fading coefficients of the two-hops are generated according to (6) and (7).

\section{Simulation Results}

We present the BER simulations of two-hop DS-CDMA systems with AF relaying technique for three different cases in this section. All simulation curves are plotted versus average SNR per hop. In the considered system, the number of simultaneous users is assumed $K=4$ and Hadamard spreading codes are used for spreading with length of $N=32$. In addition to these assumptions, all performance results are shown by varying fading parameters and number of relays using binary phase shift keying (BPSK).

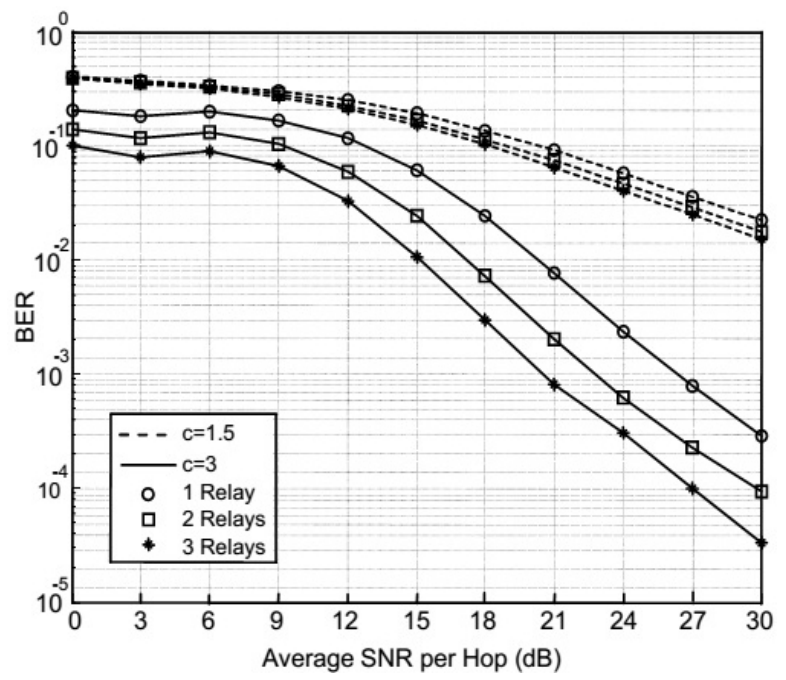

Fig. 4. BER performance of two-hop DS-CDMA with the use of AF relaying over Weibull symmetric channels using BPSK signalling.

In Fig. 4, the BER performance of DS-CDMA system 
with two-hop AF relaying is shown for the symmetric Weibull fading channels (scenario 1) in which $S-R$ and $R-D$ links are Weibull distributed. Weibull distribution includes Rayleigh fading (non LoS conditions) as a special case when $c=2$. In this figure, performance curves are obtained for different values of fading parameter for $c=1.5$ and $c=3$. From Fig. 4 , it is obvious that the performance is improved as the value of $c$ parameter is increased, as expected.

When the channel fading parameter value is $c=1.5$, the performance is slightly improved as the number of relays are increased if we compare the case of $c=3$. It can be clearly observed that increasing the number of relays has a great effect on the performance of proposed system when the fading parameter equals to 3 . For instance, when $c=1.5$, the BER values at $15 \mathrm{~dB}$ are equal to $1.9 \times 10^{-1}$ and $1.5 \times 10^{-1}$ for $L=1$ and $L=3$, respectively. In contrast to these results, if $c$ is increased to 3 , the BER values are reduced to $6,1 \times 10^{-2}$ with $L=1$ and $1 \times 10^{-2}$ with $L=3$ at $15 \mathrm{~dB}$.

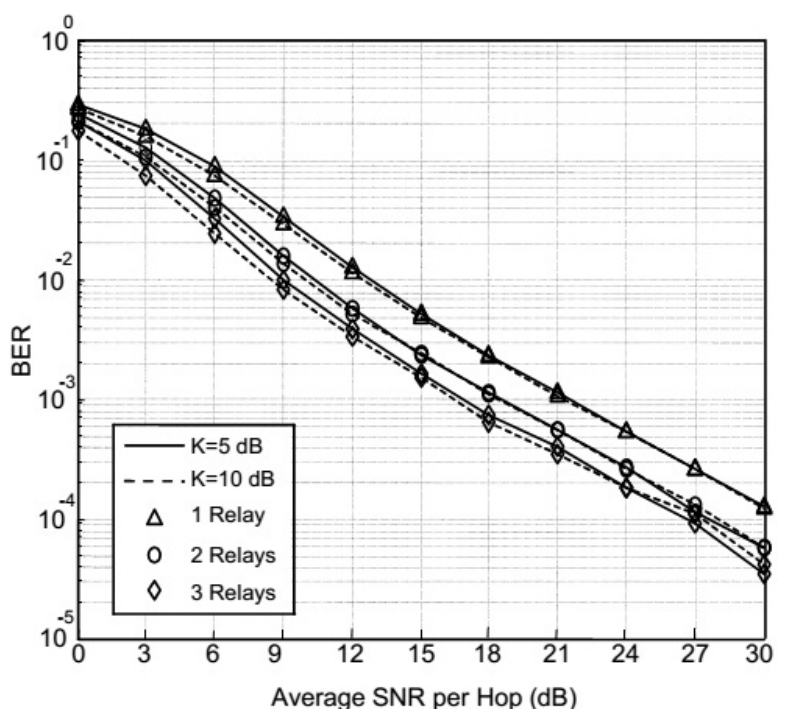

Fig. 5. BER performance of two-hop DS-CDMA using AF relaying over Rayleigh/Rician asymmetric faded channels with BPSK modulation.

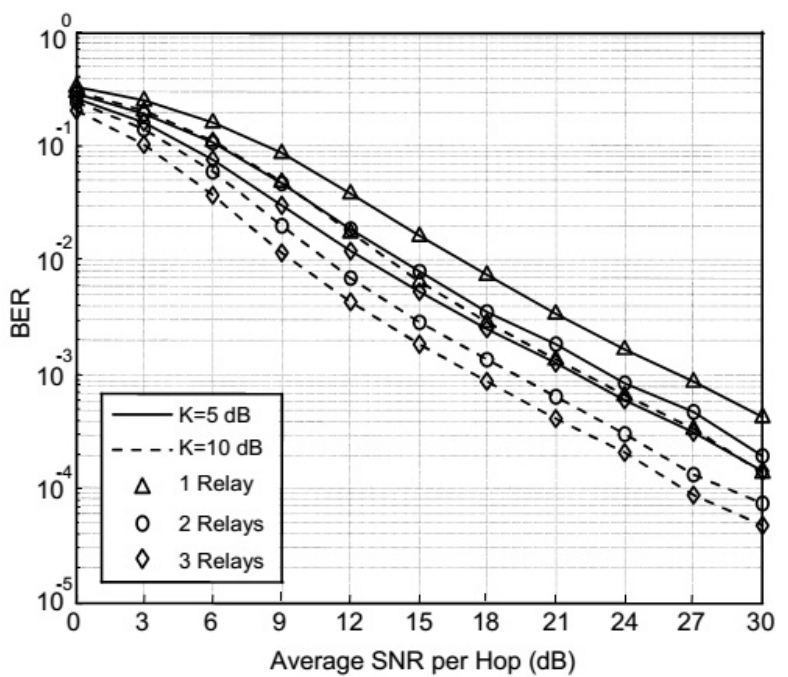

Fig. 6. BER performance of two-hop DS-CDMA using AF relaying over Rician/Rayleigh asymmetric fading channels with BPSK modulation.

In Fig. 5, the BER of the considered system model where $S-R / R-D$ links experience Rayleigh/Rician (scenario 2) fading is investigated by varying the Rician $K$ factor as $K=5 \mathrm{~dB}$ and $K=10 \mathrm{~dB}$. Figure 5 confirms that if the first hop experiences Rayleigh fading, increasing the number of relays and higher value of $K$ factor do not improve the performance for considerable amount. For example, in the case of $L=1$, the performance is almost same after $10 \mathrm{~dB}$ when $K=5 \mathrm{~dB}$ and $K=10 \mathrm{~dB}$, respectively. This observation is also valid for the cases of $L=2$ and $L=3$.

Figure 6 shows the BER of the considered system model over Rician/Rayleigh (scenario 3) asymmetric fading channels. In contrast to the results in Fig. 5, the performance is improved when the Rician $K$ factor is increased as can be seen in Fig. 6. As an example, the BER value is $7.9 \times 10^{-3}$ for the case of two relays and $K=5 \mathrm{~dB}$ at $15 \mathrm{~dB}$. However, if $K$ is increased to $10 \mathrm{~dB}$, the BER value is reduced to $2.8 \times 10^{-3}$ with two relays at $15 \mathrm{~dB}$. In scenario 3 , the amplification factor is calculated according to Rician fading (LoS conditions) coefficients so the performance is improved considerably as the number of relay nodes and Rician $K$ factor are increased.

\section{CONCLUSIONS}

We investigated two-hop DS-CDMA system in which AF method is deployed at the relay nodes over different wireless channel conditions for $S-R / R-D$ links such as Weibull/Weibull, Rayleigh/Rician and Rician/Rayleigh fading conditions. Focusing on the simulation results, some useful issues were revealed. It was demonstrated for Weibull/Weibull channels, if channel conditions are worse than non LoS Rayleigh fading $(c=1.5)$, increasing the number of relays in the system does not bring a considerable amount of performance improvement. Particularly, simulation results for Rayleigh/Rician asymmetric case illustrate that the system performance is almost same from medium SNR to high SNR range even if the value of $K$ factor is increased. Moreover, increasing the value of Rician $K$ factor exhibits considerable performance improvement when $S$ - $R$ link is distributed according to LoS Rician fading conditions as compared to the case of $S-R$ link is non LoS Rayleigh distributed which was confirmed by the simulation results. This is due to the fact that the system performance depends on the quality of $S-R$ channel in AF relaying method.

\section{REFERENCES}

[1] S. Moshavi, "Multi-user detection for DS-CDMA communications", IEEE Communications Magazine, vol. 34, no. 10, pp. 124-136, 1996. [Online]. Available: http://dx.doi.org/10.1109/35.544334

[2] C. Ciftlikli, I. Develi, "A simple and useful approach for the determination process of the weighted despreading sequences in a DS-CDMA system", European Trans. on Telecommunications, vol. 14, no. 4, pp. 361-366, 2003. [Online]. Available: http://dx.doi.org/ 10.1002/ett.4460140408

[3] A. Litvinenko, E. Bekeris, "Probability distribution of multiple-access interference in chaotic spreading codes based on DS-CDMA communication system", Elektronika ir Elektrotechnika, vol. 123, no. 7, pp. 87-90, 2012. [Online]. Available: http://dx.doi.org/ 10.5755/j01.eee.123.7.2380

[4] I. Develi, "Detection of optimal spreading codes for DS-CDMA wireless systems with despreading sequences weighted by adjustable chip waveforms", Journal of the Franklin Institute, vol. 342, pp. 69 
84, 2005. [Online]. Available: http://dx.doi.org/10.1016/j.jfranklin. 2004.08.003

[5] S. Inoshita, H. Miyazaki, F. Adachi, "Complexity-reduced perantenna multiple access interference cancellation for DAN using DSCDMA", in Proc. IEEE 80th Vehicular Technology Conf. (VTC Fall), Vancouver, Canada, 2014, pp. 1-5. [Online]. Available: http://dx.doi.org/10.1109/vtcfall.2014.6965912

[6] S. D. Gupta, S. Shomaji, F. Islam, T. Hasan, Z. Ahmed, "Performance analysis of DS-CDMA wireless communication system with and without diversity", in Proc. 16th Int. Conf. Computer and Information Technology (ICCIT), Khulna, Bangladesh, 2013, pp. $166-171$.

[7] J. N. Laneman, D. N. C. Tse, G. W. Wornell, "Cooperative diversity in wireless networks: Efficient protocols and outage behavior", IEEE Trans. Inf. Theory, vol. 50, no. 12, pp. 3062-3080, 2004. [Online]. Available: http://dx.doi.org/10.1109/TIT.2004.838089

[8] H. Boujemaa, "Exact and asymptotic BEP of cooperative DS-CDMA systems using decode and forward relaying in the presence of multipath propagation", IEEE Trans. Wireless Commun., vol. 8, no. 9, pp. 4464-4469, 2009. [Online]. Available: http://dx.doi.org/10.1109/TWC.2009.081672

[9] J. Gu, R. C. de Lamare, "Joint SIC and multi-relay selection algorithms for cooperative DS-CDMA systems", in Proc. of the 22nd European Signal Processing Conf. (EUSIPCO), Lisbon, Portugal, 2014, pp. 556-560.

[10] L. Li, L. Wang, L. Hanzo, "Successive AF/DF relaying in the cooperative DS-CDMA uplink: Capacity analysis and its system architecture", IEEE Trans. Vehicular Tech., vol. 62 , no. 2, pp. 655 666, 2013. [Online]. Available: http://dx.doi.org/10.1109/TVT.2012. 2227855

[11] M. T. Abdizadeh, A. Jafarnia, "Multiuser power allocation in downlink of cooperative CDMA networks", in Proc. 20th Int. Symposium on Personal, Indoor and Mobile Radio Communications, Tokyo, Japan, 2009, pp. 546-550. [Online]. Available: http://dx.doi.org/10.1109/pimrc.2009.5450200

[12] L. Venturino, X. Wang, M. Lops, "Multiuser detection for cooperative networks and performance analysis", IEEE Trans. Signal Process., vol. 54, no. 9, pp. 3315-3329, 2006. [Online]. Available: http://dx.doi.org/10.1109/TSP.2006.877646

[13] W. Fang, L.-L. Yang, L. Hanzo, "Transmitter-preprocessing-assisted cooperative downlink transmission in DS-CDMA systems experiencing propagation path loss and Nakagami- $m$ fading", IEEE Trans. on Vehicular Tech., vol. 58, no. 8, pp. 4182-4192, 2009. [Online]. Available: http://dx.doi.org/10.1109/TVT.2009.2021598
[14] A. Mehemed, W. Hamouda, "AF cooperative CDMA outage probability analysis in Nakagami- $m$ fading channels", IEEE Trans. Vehicular Tech., vol. 62, no. 3, pp. 1169-1176, 2013. [Online]. Available: http://dx.doi.org/10.1109/TVT.2012.2227576

[15] A. Eid, W. Hamouda, I. Dayoub, "Performance of multi-relay coded cooperative diversity in asynchronous code-division multiple-access over fading channels", IET Commun., vol. 5, no. 5, pp. 683-692, 2011. [Online]. Available: http://dx.doi.org/10.1049/ietcom.2010.0478

[16] H. A. Suraweera, G. K. Karagiannidis, P. J. Smith, "Performance analysis of the dual-hop asymmetric fading channel", IEEE Trans. Wireless Commun., vol. 8, no. 6, pp. 2783-2788, 2009. [Online]. Available: http://dx.doi.org/10.1109/TWC.2009.080420

[17] J. Ouyang, M. Lin, Y. Zhuang, "Performance analysis of beamforming with feedback delay in two-hop AF relaying over Rayleigh-Rician fading channels", Electron. Letts., vol. 48, no. 11 , pp. 663-664, 2012. [Online]. Available: http://dx.doi.org/10.1049/el. 2012.0790

[18] W. Xu, J. Zhang, P. Zhang, "Performance analysis of dual-hop amplify-and-forward relay system in mixed Nakagami- $m$ and Rician fading channels", Electron. Letts., vol. 46, no. 17, pp. 1231-1232, 2010. [Online]. Available: http://dx.doi.org/10.1049/el.2010.1555

[19] N. Kapucu, M. Bilim, I. Develi, "Outage probability analysis of dualhop decode-and-forward relaying over mixed Rayleigh and generalized Gamma fading channels", Wireless Pers. Commun., vol. 71, no. 2, pp. 947-954, 2013. [Online]. Available: http://dx.doi.org/10.1007/s11277-012-0853-6

[20] P. Spalevic, M. Stefanovic, S. Panic, S. Minic, L. Spalevic, "Amplify-and-forward relay transmission system over mixed Rayleigh and Hoyt fading channels", Elektronika ir Elektrotechnika, vol. 120, no. 4, pp. 21-25, 2012. [Online]. Available: http://dx.doi.org/10.5755/j01.eee.120.4.1445

[21] N. Kapucu, M. Bilim, I. Develi, Y. Kabalci, "Performance of two-hop relay assisted decode-and-forward transmission under mixed fading environments", Elektronika ir Elektrotechnika, vol. 21, no. 1, pp. 6063, 2015. [Online]. Available: http://dx.doi.org/10.5755/j01. eee.21.1.9806

[22] Y. Lee, M.-H. Tsai, "Performance of decode-and-forward cooperative communications over Nakagami- $m$ fading channels", IEEE Trans. on Vehicular Tech., vol. 58, no. 3, pp. 1218-1228, 2009. [Online]. Available: http://dx.doi.org/10.1109/TVT.2008.928907

[23] M. K. Simon, M.-S. Alouini, Digital communication over fading channels. New York: Wiley, 2000.[Online]. Available: http://dx.doi.org/10.1002/0471200697 